Außer diesen gibt es noch andere unlösliche Lokalanästhetica, die Orthoformgruppe, welche außer dem Orthoform, das Anästhesin, und seit der neuesten Zeit das vom Vortr. erfundene noch stärkere Propäs i n (Paramidobenzoesäurepropylester)

$$
=\mathrm{C}_{6} \mathrm{H}_{4}<\underbrace{\mathrm{NH}_{2}}_{\mathrm{COOC}_{3} \mathrm{H}_{7}}
$$

angehören, welches sich als das bestwirkende aus einer ganzen Anzahl vom Redner dargestellten alten und neuen Verbindungen erwies. Diese Anästhetica unterscheiden sich vom Cocain durch noch größere Ungiftigkeit, so daß sie auch innerlich bei Magenkrankheiten eingenommen werden können, sind aber andererseits unlöslich in Wasser, so daß sie weder zu Injektionen, noch zu Rücken markanästhesie anwendbar sind, sonst aber auf fast allen Gebieten der Medizin in Verwendung gezogen werden können. Auch diese Präparate zeigen die für Lokalanästhetica erwähnten Gesetzmäßigkeiten wie das Vorhandensein des Benzoesäureradikals, der Amidogruppe usw.

Die SchluBbetrachtung war dem gegen die mörderische Schlafkrankheit dienenden Mittel Atoxyl (aminophenylarsinsaures Natrium) gewidmet, dessen Studium und Vervollkommnung darch chemisch ähnliche Körper besonders von $\mathrm{E} \mathrm{h} \mathrm{r} \mathrm{li} \mathrm{ch}$, dem Leiter des Instituts für experimentelle Therapie und Infektionskrankheiten zu Frankfurt a. M., eifrig betrieben wird. Dieser Forscher fand auch, daß gewisse organische Farbstoffe, wie das Methylenblau, das Neutralrot usw. eine Verwandtschaft zu gewissen organischen Geweben besitzen und nur diese färben. Durch diese Tatsachen sind der Chemotherapie ganz neue Wege gewiesen.

So ist zu hoffen, daß die Pharmakologie zum Heile der Menschheit weiter fortschreiten wird. Deutschland nimmt heute den ersten Platz in dieser Wissenschaft ein. Dies hat unser Vaterland in erster Linie der hohen Entwicklungsstufe seiner chemischen Wissenschaft und seiner chemischen Industrie zu verdanken.

\section{Chemische Industrie auf der Wiesbadener Ausstellung 1909.}

\author{
Von A. Clourir. \\ (Eingeg, d. 27. 7. 1909.)
}

Auf der Ausstellung für Handwerk und Gewerbe, Kunst und Gartenbau in Wiesbaden ist verschiedenes zu sehen, was auch die Leser dieser Zeitschrift interessieren dürfte.

Die Ausstellung der bekannten Ingenieurfirma E. Hartmann \& F. Benker, Wiesbaden-Paris, ist in d. Z. schon auf S. 953 erwähnt. Hier sei nur noch auf das Modell einer vollständigen Schwefelsäurekonzentrationsanlage, nach eigenem System der Aussteller, das durch Wiedergabe einzelner Teile dieses Apparates weiter verdeutlicht ist, hingewiesen.
Bekanntlich konzentriert die Firma in Schalen aus sogen. Neutraleisen von großer Widerstandsfähigkeit.

Von Interesse ist ferner der sogen. "Stellakitt" des Stellawerkes Homburg a. Rh. Derselbe bildet ein absolut säurebeständiges Bindemittel für Schamottematerial und widersteht dauernd selbst den Einflüssen heißer Salpetersäuredämpfe. Dieses Material ist in verschiedenen Gefäßen gezeigt, die in Säuren eingesetzt sind. - Erwähnenswert ist auch der vom Tonwerk Biebrich ausgestellte komplette Retortenofen. Neben diesem das Modell einer neuen, transportablen, auseinandernehmbaren, drehbaren Brennanlage, welche einen rationellen, ununterbrochenen Betrieb für Ziegeleien, Tonwarenfabriken usw. ermöghicht, hergestellt von W. Berghäuser und Jos. Gullich, Keramiker, Biebrich a. Rh. - Die Chem. Fabrik ,,Elektro", G. m. b. H., Biebrich a. Rh. und Lyon, zeigt die nach eigenem, patentierten Verfahren hergestellten Stanzblöcke aus präpariertem Papierstoff für Kartonnage- und Schuhfabriken usw.. ferner patentierte Hackklötze aus präpariertem Papierstoff und Parkettböden aus diesem Material. - Kraenkel \& Schallbruch, Wiesbaden führen ihre Erfindung vor: Eine Walzenformmaschine mit Handbetrieb zur Herstellung von Zementwanddielen, welche den Vorteil besitzt, daß das bisherige zeitraubende und anstrengende Einstampfen der Zementdielen in einen Rahmen vermieden werden kann. -- Beachtung verdient die Ausstellung der Wunnerschen Bitumen-Werke, G. m. b. H., Chemische Fabrik Unna i. W., mit ihrer Wunnerschen Bitumenemulsion und Ceresit. in der Anwendung. Diese Präparate dienen dazu, den gewöhnlichen Zement, Zementmörtel und Sand völlig wasserdicht zu machen. Man sieht dies an den ausgestellten Zylindern, die mit Wasser gefüllt sind. Dieser Wasserdruck wirkt auf eine $50 \times 50 \mathrm{~cm}$ grole und $2,5 \mathrm{~cm}$ starke Zementplatte ein. Letztere in der Mischung von 1 Teil Zement, 3 Teile Sand und etwa 1,5\% Ceresitzusatz. Die Platte bleibt trotz des hohen Wasserdrucks vollkommen trocken.

In einer anderen Abteilung der Ausstellung, welche die für den Weinbau in Betracht kommenden Gegenstände aufweist, dürfte für uns die technische Abteilung des Hofrat Dr. Schmittschen Laboratoriums, Wiesbaden, von besonderem Interesse sein. Zunächst sehen wir da Apparate, Gefäße und Material zur Herstellung und zum Versand von Reinzuchthefen für die Bereitung von Trauben-, Obstund Schaumwein. Über 50 verschiedene sterilisierte, in kleinen Champagnerflaschen befindliche und den hervorragendsten Weinbaugebieten des deut schen Reiches entstammende Mostproben, aus denen im Herbst 1908 neue Heferassen gezüchtet worden sind, legen Zeugnis ab von der regen Tätigkeit des Laboratoriums, die bereits mehrere 100 Rassen enthaltende Sammlung zu vermehren. Die Herstellung der Reinhefe für den Versand erfolgt in eigenartigen Flaschen von 8-15 I Inhalt, die die Form von Abklärgefäßen und Wou If $f$ schen Flaschen besitzen. Sie sind am Boden mit Tubus und eingeschliffenen, vor Infektion geschützten Hähnen versehen und enthalten in den Hälsen Gasröhren und mit steriler Watte gefüllte Luftdurchlaßröhren, so daß sie zugleich zum Vergären von Most und zum Abfüllen der flüssigen Reinhefe die- 
nen und längere Zeit benutzt werden können. Neben diesen Apparaten fallen die Flaschen zum Sterilisieren von Flüssigkeiten und festen Substanzen, wie Most, Milch, Obst usw. ins Auge. Diese Apparate beruhen im Gegensatz zu dem bei sämtlichen Systemen von Einmachgläsern beobachteten Prinzip darauf, daß nicht wie bei diesen die Luft nach erfolgter Sterilisierung möglichst abgehaiten wird, sondern sie ermöglichen vielmehr den Zutritt der Luft durch die an den aufgeschliffenen Kappen befindlichen, mit Watte gefüllten Kugeln, die hierdurch filtriert, also keimfrei gemacht wird. — Sehr interessant ist die reichhaltige Sammlung von Weinen, welche die hauptsächlichsten Krankheiten und Fehler derselben zum Ausdruck bringt. Erläutert werden diese durch eine Anzahl mikroskopischer Skizzen, welche das Laboratorium bei gerichtlichen Untersuchungen von Weinen anfertigt und den chemischen Gutachten beifügt. - Außer diesen speziell die Weintechnik betreffenden Gegenständen sehen wir noch eine Anzahl von chemischen Präparaten. Zuerst die verschiedenartigen Nährböden, wie solche von Laboratorien für bakteriologische und gärungsphysiologische Zwecke verwendet werden. Da sind Fleischwasser- und Fleischextrakt-, Peptongelatinen, Milchserum- und verschiedene Agar-Agarnährböden, Most- und Weingelatinen u. dgl. in Reagierröhrchen und eigens für solche Zwecke konstruierten Abschmelz- und Versandflaschen. In sogen. $\mathrm{P}$ e $\mathrm{t} r$ i schen Schalen, die mit den verschiedensten Nährböden versehen sind, wird das Wachstum der Bakterien von Lebensmitteln veranschaulicht, wodurch dem Laienpublikum Gelegenheit geboten wird, die Anwendung dieser Nährböden bei der Untersuchung von Nahrungs- und Genußmitteln kennen zu lernen.

\section{Referate.}

\section{2. Analytische Chemie, Labora- toriumsapparate und allgemeine Laboratoriumsverfahren.}

Ath. J. Sofianopoulos. Direkte Bestimmung zusammen vorkommender Körper ohne Trennung. (Bll. Soc. chim. [4] 5, 632-641. Juni 1909. Athen.)

Die Methode beschränkt die Zahl der Wägungen und Filtrationen auf ein Minimum. An dem praktischen Beispiel des Pyrolusits wird sie näher erläutert. Zunächst wird die durch Behandlung des Materials mit Salzsäure abgeschiedene Kieselsäure gewogen. Die salzsaure Lösung enthält Eisen, Aluminium, Mangan, ferner Calcium, Barium und Magnesium. In aliquoten Teilen werden einerseits Eisen, Aluminium und Mangan zusammen als Oxyde bestimmt und dann in die Fluoride übergeführt and als solche wieder gewogen; andererseits wird das Mangan durch Titration mit Permanganat für sich bestimmt. Aus den erhaltenen Gewichten lassen sich dann die Werte für Eisen und Aluminium berechnen. Analog werden CaIcium, Barium und Magnesium zusammen als Oxyde und als Fluoride gewogen, und dann das Barium für sich als Silicofluorid bestimmt. Aus den gefundenen Gewichten lassen sich die Werte für Calcium und Magnesium berechnen. - Die Berechnung beruht auf komplizierten Gleichungen, die durch mathematische Deduktionen erhalten werden.

$$
\text { Wr. [R. 2460.] }
$$

\section{L. De Koninck. Anwendung von Eisenalaun als} Urtitersubstanz in der Mabanalyse. (Bll. Soc. chim. Belg. 23, 222--225. [Febr.] Mai 1909. Lüttich.)

Verf. hält das Ferriammoniumsulfat $\left[\left(\mathrm{NH}_{4}\right)_{\mathbf{2}} \mathrm{Fe}_{\mathbf{2}}\right.$ $\left.\left(\mathrm{SO}_{4}\right)_{4} \cdot 24 \mathrm{H}_{2} \mathrm{O}\right]$ für hervorragend geeignet als Urtitersubstanz. Das Handelsprodukt muß durch Umkrystallisieren gereinigt werden. Nachdem man die Krystalle eine halbe Stunde lang auf Filtrierpapier hat liegen lassen, werden diese in einer gut verschlossenen Flasche aufbewahrt, wo sie sich unbegrenzte Zeit halten. Vor dem Abwägen zur
Verwendung muß man die Krystalle etwa eine Viertelstunde bei geöffnetem Wagekasten offen auf der Wage liegen lassen. Nach dieser Zeit ist die letzte den Krystallen noch anhaftende Feuchtigkeit verdunstet, und das Gewicht ändert sich innerhalb dreiviertel Stunden nicht mehr.

$$
\text { Wr. [R. 2459.] }
$$

L. Birkenbach. Die Untersuchungsmethoden des Wasserstoffsuperoxyds. (Chem. Analyse $\gamma, 1$ bis 142. Mai 1909. Ludwigshafen a. Rh.)

Verf. hat in der umfangreichen Arbeit den gesamten analytischen Stoff der Fuchliteratur auf dem Gebiete des Wasserstoffsuperoxyds gesammelt und im Sinne der gegenwärtigen Anschauungen der wissenschaftlichen Chemie kritisch gesichtet. Nach zwei allgemeinen Kapiteln: 1. Historisches, Terminologisches und Theoretisches über Wasserstoffsuperoxyd und 2. Herstellung, Eigenschaften und Verwendung des Wasserstoffsuperoxyds bespricht er zunächst die verschiedenen Methoden zum qualitativen Nachweis des Wasserstoffsuperoxyds. Von den hierzu benutzten 21 Reaktionen, die er am Schlusse der Besprechung in einer Tabelle zusammenstellt, bezeichnet er die Reaktion mit 'Titansäure als die charakteristischeste und zuverlässigste für Wasserstoffsuperoxyd, während er bezüglich der Empfindlichkeit den Nachweis mittels Guajactinktur-Malzauszug obenan stellt. - Von den Methoden zur quantitativen Bestimmung des Wasserstoffsuperoxyds ist nach Ansicht B i r k e n b a c h s die Kaliumpermanganatmethode die bewährteste und von hervorragender Genauigkeit. Die quantitativen Methoden sind gleichfalls am Schlusse der Besprechung in einer Tabelle zusammengestellt.

$$
\text { Wr. [R. 2466.] }
$$

F. A. Gooch und H. L. Ward. Die Fällung des Kupfers als Kupferoxalat. (Z. anorg. Chem. 6z, 348-359. [April] Juni 1909. Neu-Haven U.S. A.) (Aus dem Am. Journ. sci. ins Deutsche übertragen von $\mathbf{F}$. $\mathbf{K}$ oh $\mathbf{n}$ - Berlin.)

Verf. hat die von Peters (Am. Journ. sci. 10, 359) angegebene Methode einer eingehenden Prüfung unterzogen. Als bequemstes und bestes Ver- 